\section{The Need for a Low-Expense Universally Acceptable rhTSH Protocol}

TO THE EDITOR: Thyroid cancer is a rare malignancy (1). Most cases are of well-differentiated epithelial papillary and follicular carcinomas. As most thyroid cancer has a low mortality rate and a moderately high recurrence rate, lifelong monitoring is required $(2,3)$. The 2 most important diagnostic tools for monitoring are serum thyroglobulin and whole-body radioiodine scans. They have been shown to be more accurate when performed after thyroid-stimulating hormone (TSH) stimulation.

Endogenous TSH stimulation by thyroid hormone withdrawal causes symptoms of hypothyroidism and impairs a patient's healthrelated quality of life (4). Recombinant human TSH (rhTSH) can produce similar TSH stimulation while the patient remains euthyroid on thyroid hormone replacement (5). The sensitivity for disease detection by radioiodine scanning and serum thyroglobulin measurements after rhTSH stimulation is comparable to that of the conventional thyroid hormone withdrawal method (6). Because symptoms of hypothyroidism are avoided, these patients do not experience a decrease in quality of life and maintain their ability to work, thus reducing economic loss for the payer and society (7). Moreover, patients treated with rhTSH are less likely to need sick leave than those whose thyroxine is withdrawn (8).

Cost-effectiveness studies have shown that rhTSH-treated patients have a better quality of life, return to work earlier, and make less use of the health care system. These observations might stand true in the developed world, but in developing countries such as India, the per capita income is low (cost of rhTSH per patient in India, \$1,200, annual per capita income in India, $\sim \$ 924.91$ ) and medical reimbursement is not freely available. Therefore, rhTSH administered by the standard method is beyond the reach of most patients.

In our department, we have found that patients usually do not show symptoms of hypothyroidism until up to 2-3 wk. Therefore, we hypothesize that a combined protocol (withdrawal for $15 \mathrm{~d}$ and a single dose of rhTSH) may substantially reduce the cost and will be only slightly less if not equally effective.

Reviewing the literature, we found a study by Pacini et al. that assessed whether rhTSH stimulation may be used in patients with differentiated thyroid carcinoma for postsurgical ablation of thyroid remnants using a $1,110-\mathrm{MBq}(30-\mathrm{mCi})$ standard dose of ${ }^{131} \mathrm{I}$ during thyroid hormone therapy (9). The authors compared 3 treatment arms: in the first, patients were treated while hypothyroid; in the second, patients were treated while hypothyroid and were stimulated in addition with rhTSH (hypothyroid + rhTSH); in the third, patients were treated while euthyroid on thyroid hormone therapy and were stimulated with rhTSH (euthyroid + rhTSH). In the first 2 arms, patients were rendered hypothyroid by withdrawal of L-thyroxine therapy $45 \mathrm{~d}$ before ablation and of L-triiodothyronine $15 \mathrm{~d}$ before ablation. The rate of successful ablation was similar in the hypothyroid and hypothyroid + rhTSH arms ( $84 \%$ and $78.5 \%$, respectively). A significantly lower rate of ablation (54\%) was achieved in the euthyroid + rhTSH arm. The authors concluded that, using stimulation with rhTSH, a 1,110-MBq standard dose of radioiodine is not sufficient for a satisfactory thyroid ablation rate. Another important observa-

COPYRIGHT ( 2011 by the Society of Nuclear Medicine, Inc. tion of this study was that the euthyroid + rhTSH arm had low TSH, low 24-h radioiodine uptake, a low initial dose rate, and accelerated iodine clearance in comparison with the other 2 arms. The authors suggested that possible alternatives for obtaining a satisfactory rate of thyroid ablation with rhTSH may consist of increasing the dose of radioiodine or using different protocols of rhTSH administration to produce more prolonged thyroid cell stimulation. However, the authors used hormone withdrawal and rhTSH (hypothyroid + rhTSH) in the second arm, and this choice may lead to symptomatic hypothyroidism and the additional cost of $2 \mathrm{rhTSH}$ injections.

Therefore, we put forward a shortened, 15-d, protocol (withdrawal for $15 \mathrm{~d}$ and a single dose of rhTSH) that may be a good alternative to other costlier or uncomfortable protocols. In this new protocol, the problems associated with the euthyroid + rhTSH method, namely low TSH level, low 24-h radioiodine uptake, and rapid clearance of ${ }^{131}$ I, may be reduced. Moreover, the patient will not have symptoms of hypothyroidism at $2 \mathrm{wk}$ after stopping L-thyroxine. A single dose of rhTSH reduces the cost by half, in comparison to the standard rhTSH preparation method with 2 doses.

A randomized controlled trial is needed to further clarify whether this hypothesis is true. If successful, the new protocol may reduce the cost of thyroid cancer management substantially.

\section{REFERENCES}

1. What are the key statistics for thyroid cancer? American Cancer Society Web site. Available at: http://www.cancer.org/Cancer/ThyroidCancer/DetailedGuide/thyroidcancer-key-statistics. Published May 12, 2010. Updated April 7, 2011. Accessed May 19, 2011.

2. Mazzaferri EL, Jhiang SM. Long term impact of initial surgical and medical therapy on papillary and follicular thyroid cancer. Am J Med. 1994;97:418-428.

3. Sherman SI, Brierley JD, Sperling M, et al. Prospective multicenter study of thyroid carcinoma treatment: initial analysis of staging and outcome. Cancer: 1998;83:1012-1021.

4. Dow KH, Ferrell BR, Anello C. Quality of life changes in patients with thyroid cancer after withdrawal of thyroid hormone therapy. Thyroid. 1997;7:613-619.

5. Meier CA, Braverman LE, Ebner SA, et al. Diagnostic use of recombinant human thyrotropin in patients with thyroid carcinoma (phase I/II study). J Clin Endocrinol Metab. 1994;78:188-196.

6. Haugen BR, Pacini F, Reiners C, et al. A comparison of recombinant human thyrotropin and thyroid hormone withdrawal for the detection of thyroid remnant or cancer. J Clin Endocrinol Metab. 1999;84:3877-3885.

7. Luster M, Felbinger R, Dietlein M, Reiners C. Thyroid hormone withdrawal in patients with differentiated thyroid carcinoma: a one hundred thirty-patient pilot survey on consequences of hypothyroidism and a pharmacoeconomic comparison to recombinant thyrotropin administration. Thyroid. 2005;15:1147-1155.

8. Borget I, Corone C, Nocaudie M, et al. Sick leave for follow-up control in thyroid cancer patients: comparison between stimulation with Thyrogen and thyroid hormone withdrawal. Eur J Endocrinol. 2007;156:531-538.

9. Pacini F, Molinaro E, Castagna MG, et al. Ablation of thyroid residues with $30 \mathrm{mCi}$ ${ }^{131} \mathrm{I}$ : a comparison in thyroid cancer patients prepared with recombinant human TSH or thyroid hormone withdrawal. J Clin Endocrinol Metab. 2002;87:4063-4068.

\section{Manish Ora* \\ Madhusudanan P \\ Suruchi Jain \\ Sukanta Barai \\ Sanjay Gambhir \\ *Department of Nuclear Medicine \\ SGPGI, Rae-Barelli Rd. \\ Lucknow, UP, India 226014 \\ E-mail:drmanishora@yahoo.com}

DOI: 10.2967/jnumed.110.081174 